\title{
Multi-robot Surveillance: an Improved Algorithm for the GRAPH-CLEAR Problem
}

\author{
Andreas Kolling Stefano Carpin \\ School of Engineering \\ University of California, Merced \\ Merced - USA
}

\begin{abstract}
The main contribution of this paper is an improved algorithm for the GRAPH-CLEAR problem, a novel NP-complete graph theoretic problem we recently introduced as a tool to model multi-robot surveillance tasks. The proposed algorithm combines two previously developed solving techniques and produces strategies that require less robots to be executed. We provide a theoretical framework useful to identify the conditions for the existence of an optimal solution under special circumstances, and a set of mathematical tools characterizing the problem being studied. Finally we also identify a set of open questions deserving more investigations.
\end{abstract}

\section{INTRODUCTION}

The use of multi-robot systems for the surveillance of vast regions is one of the well established areas in multi-robot research. Up to now, however, there have been still very few on-field deployments of these systems for real world applications. Besides the obvious matter of cost, another reason for their moderate use is the fact that many basic questions about the efficient coordination of these systems are still unanswered. A big fraction of former theoretical research developed models where robots were equipped with sensors abstractions pretty far from realistic applications, e.g. sensors with infinite range and the alike. In this paper we instead extend our previous findings aimed to investigate surveillance tasks by multi-robot systems where individual agents use sensors with limited capabilities. We started this research thread with two papers [5][6] aimed to extend the CMOMMT (Cooperative Multi-robot Observation of Multiple Moving Targets) problem initially posed by Parker [11]. One of the main limitations of these algorithms is the requirement that robots operate in open areas. Our following efforts have therefore been devoted to scenarios where robots operate in cluttered environments [7]. In particular, we modeled the problem of discovering multiple intruders in a complex environment using a novel graph theoretic problem, dubbed GRAPH-CLEAR. Informally speaking, the problem asks what is the minimum number of robots needed to detect all possible intruders in a given complex environment that can be modeled as a graph. In [8] we proved that the associated decision problem is NP-complete. As clarified later on, a way to circumvent the intractability of the problem on graphs, is to perform certain guard operations that turn graphs into trees. In [7] and [8] we have provided two algorithms that produce search strategies for trees, i.e. course of actions for a robot team that ensures each intruder will be discovered. Both algorithms are known to be suboptimal. In this paper we present a new approach for the GRAPH-CLEAR problem restricted to trees that outperforms the previous ones. It is worth to outline that many of the properties regarding the GRAPH-CLEAR problem restricted to trees are still to be investigated. For example, we do not know yet whether such restriction to trees allows to find the optimal solution in polynomial time. This paper, however, provides a further improvement that sheds some more light on this problem, and provides some more formalism that could be used to answer this question and similar ones.

The paper is organized as follows. In section II we revise former research related to multi-robot surveillance, and we provide references to seminal papers on graph theory related to the problem at hand. Section III summarizes the GRAPH-CLEAR problem and shortly addresses our formerly developed algorithms. The new approach and a theoretical framework are presented in section IV, followed by a new algorithm that computes strategies for the new approach under certain conditions. Section V concludes with a discussion of remaining problems and possible extensions of the presented work.

\section{RELATED RESEARCH}

Visibility-based pursuit evasion games have attracted remarkable attention from the robotics community. On the theoretical side Suzuki and Yamashita first investigated the problem of a pursuer searching intruders using a beam sensor with unlimited range [14]. LaValle and colleagues further investigated this problem considering various restrictions and extensions. For example, the case of an omnidirectional unlimited range sensor was investigated [4], or the case of a robot equipped just with a gap sensor, i.e. a sensor capable only of detecting discontinuities [13]. On the more applied side, the formerly cited work on CMOMMT by Parker [11] set a milestone in the field. More recently Gerkey at al. [3] describe an implementation of the visibility based pursuit evasion problem on a robot with limited field of view.

Researchers in graph theory also investigated problems related to graph search. Three papers are particularly important in order to put our contribution into context. The concepts of contaminated and clear edges were introduced by Parsons [12], who pioneered this research vein. The problem he defined, called edge-search, deals with graphs where edges can be contaminated and have to be cleared by agents placed on vertices or marching along edges. The search number 
$s(G)$ of the edge-search problem is the smallest number of agents with which one can find a sequence of actions, called strategy, such that all edges become clear. The problem of determining $s(G)$ was shown to be NP-hard by Megiddo et al. in [10]. An important extension to Parson's work was proposed by Barriere et al. [1], who first considered the edge-search problem with weighted vertices and edges. This extension implies that more than one agent is needed to perform the basic operations of clearing an edge or blocking a vertex. They also introduce the concept of contiguous strategies, i.e. solving strategies such that the clear subset of vertices always forms a connected subgraph of the original graph. They show that optimal contiguous strategies can be found in linear time on trees (contiguous strategies are however not optimal in general).

\section{GRAPH CLEAR}

This section offers a formalization of the GRAPH-CLEAR problem, pertinent notation and current algorithms for computing strategies on trees which serve as a basis for the new approach. Before moving to the formalism, we outline the connection between real world problems and the mathematical models presented herein. We are mainly interested in scenarios where robots operate in complex indoor environments with many rooms connected by multiple doors. In this scenario, rooms are modeled as graph vertices, while doors are mapped into graph edges connecting adjacent vertices (i.e. rooms). Figure 1 shows a simple environment and its corresponding graph model.
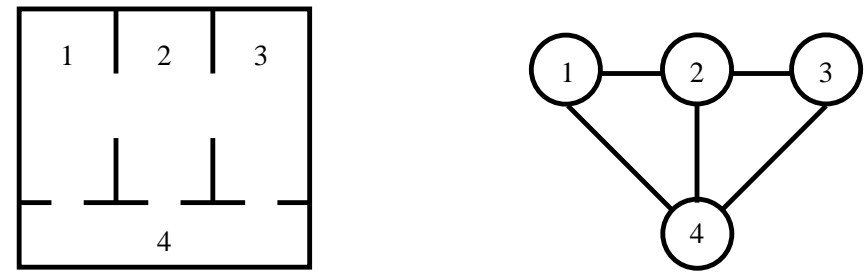

Fig. 1. An indoor environment and its corresponding graph model for the GRAPH-CLEAR problem.

As our focus is on robots with restricted capabilities, we assume that multiple robots are needed to patrol and search these environments. In particular, we suppose that in order to guarantee that no intruder crosses a door, we need to place a certain number of robots to guard it. This number is indicated as the weight of an edge. Similarly, more than one robot could be needed in order to sweep a room and make sure it contains no intruder, or detect them. This number is indicated as the weight of a vertex. While in the following we strictly stick to graph theory jargon, the reader could translate every instance of the word vertex with room, edge with door and agent with robot.

\section{A. Definitions}

GRAPH-CLEAR was formalized in [8] and is here shortly summarized. We define a weighted graph $^{1}$ as a triple $G=$

\footnotetext{
${ }^{1}$ while in graph related literature weighted graphs have weights for edges only, we instead assume that weights are defined both for edges and vertices.
}

$(V, E, w)$, where $V$ is the set of vertices, $E$ is the set of edges, and $w: V \cup E \rightarrow \mathbb{N} \backslash\{0\}$ is the weight function. The graph is undirected. Edges and vertices can be clear or contaminated. A clear vertex or edge hosts no intruders, while a contaminated vertex or edge could potentially hide one or more intruders. $G$ is said to be clear when all vertices and edges are clear. A clear vertex $v$, however, can become contaminated again if there exists a path from $v$ to another contaminated vertex or edge ${ }^{2}$. Recontamination of edges is analogue. Contaminated vertices or edges can be cleared by applying clearing and blocking operations respectively. A clearing operation applied to a vertex $v \in V$ detects all intruders under the assumption that no new intruders enter or leave the vertex. It hence clears the vertex using $w(v)$ agents. A blocking operation is applied to an edge $e \in E$ and detects all intruders passing through the edge and as such prevents contamination from spreading through the edge and clears it. The number of agents needed for a block is $w(e)$. When using multiple agents to clear a graph, we can deploy agents in edges or vertices in order to perform the blocking and clearing operations defined above. The policy we follow when deploying agents is called strategy and defined as:

Definition 1 (Strategy): Let $G=(V, E, w)$ be a weighted graph. A strategy $S$ for $G$ is a function $S:(V \cup E) \times \mathbb{N} \rightarrow \mathbb{N}$.

$S(x, t)$ is the number of agents deployed on $x \in V \cup E$ at time $t$. If $S(x, t) \geq w(x)$, then $x$ is blocked or cleared at time $t$, depending on whether it is an edge or a vertex. Associated with each strategy there is a cost, i.e. the number of agents needed in order to implement the strategy.

Definition 2 (Cost of a strategy): Let $G=(V, E, w)$ be as before and let $S$ be a strategy for $G$. The cost of $S$ is

$$
a g(S)=\max _{t \in \mathbb{N}} \sum_{x \in V \cup E} S(x, t)
$$

A strategy $S$ clears a graph $G$, if by deploying agents in the order dictated by the strategy, there exist a time $t$ such that all edges and vertices are clear. While this notion could be made formal, we here omit the details. This leads us to the GRAPH-CLEAR problem.

Definition 3 (GRAPH-CLEAR problem): Let $G$ be as before with all edges and vertices contaminated. Determine a strategy $S$ for $G$ that clears $G$ and is of minimal cost $a g(S)$. The following formula gives the cost to clear a vertex safely, i.e. the cost to perform a clearing operation on the vertex while blocking all the edges connected to it to avoid immediate recontamination.

$$
s(v):=w(v)+\sum_{e \in \operatorname{Edges}(v)} w(e) .
$$

Any strategy which clears more than one vertex at time $t$ has $a g(S) \geq a g\left(S^{\prime}\right)$ for some strategy $a g\left(S^{\prime}\right)$ which clears at most one vertex at time $t$. Therefore, as the focus of our research is in finding strategies of minimal cost, we will exclusively deal with strategies clearing at most one vertex

\footnotetext{
${ }^{2}$ we also consider edges connecting two vertices in the path, contrary to the common definition of a path as a sequence of vertices. We opt not to formalize this slight difference to keep the notation simpler.
} 
at once. There may be multiple strategies $S$ of minimal cost, so we define the number of agents to clear a graph $G$ as $a g(G):=a g(S)$ for any optimal strategy $S$.

\section{B. Previous results for GRAPH-CLEAR}

The concepts of contiguous and non-contiguous strategies play an important role in the algorithms we have formerly developed. As defined by Barriere at al. [1], a contiguous strategy requires that the subset of cleared vertices forms a connected subgraph. This requirement is relaxed for non-contiguous strategies. In [7] the GRAPH-CLEAR problem was first attacked, and an algorithm to produce non-contiguous strategies on trees was presented, as well as a upper bound on its cost w.r.t. to the depth of the tree. The algorithm is based on the computation of labels on edges which we will shortly present in this section. In [8] the NP-completeness of GRAPH-CLEAR was proven, and an algorithm to compute contiguous strategies on trees was presented. It was shown that both algorithms produce suboptimal strategies for trees. The contiguous algorithm may, however, produce optimal contiguous strategies as discussed in [8]. Since contiguousness is a rather strict requirement that is not necessary in most robotics applications we investigate non-contiguous strategies to yield a lower number of agents. In [8] it was proposed to combine the two algorithms, i.e. the one producing sub-optimal non-contiguous strategies and the one producing contiguous strategies. An approach for finding non-contiguous strategies based on the two former algorithms, its theoretical properties and an improved algorithm are the primary contributions of this paper. First, we will shortly introduce the underlying mechanisms of the two previous algorithms. We restrict the problem to trees, and let $G_{T}=(V, E, w)$ be an instance of the GRAPH-CLEAR problem with $G_{T}$ being a weighted tree. An instance of GRAPH-CLEAR on a graph can be reduced to an instance on a tree by permanently deploying a set of agents on suitable edges, so that the graph stays connected but exhibits no cycles. Since this cost is constant we will not consider it during the optimization process.

1) Non-contiguous labels: Let $v_{x}, v_{y} \in V$ and $e=$ $\left[v_{x}, v_{y}\right] \in E$. We are assigning a label $\lambda_{v_{x}}(e)$ to edge $e$ to represent the number of agents needed to clear the subtree rooted in $v_{y}$ when entering from $v_{x}$. If $v_{y}$ is a leaf, then $\lambda_{v_{x}}(e)=s\left(v_{y}\right)=w\left(v_{y}\right)+w(e)$. Otherwise consider all neighbors of $v_{y}$ other than $v_{x}$. Let these be $v_{2}, \ldots, v_{m}$ with $m=\operatorname{degree}\left(v_{y}\right)$. Write $e_{i}:=\left[v_{y}, v_{i}\right]$ and let all $v_{i}$ be ordered s.t. $\rho_{i} \geq \rho_{i+1}$ where $\rho_{i}:=\lambda_{v_{y}}\left(e_{i}\right)-w\left(e_{i}\right)$. The ordering defines the sequence in which we clear the vertices $v_{i}$. The clearing cost of the subtree rooted at $v_{i}$ is:

$$
c\left(v_{i}\right):=\lambda_{v_{y}}\left(e_{i}\right)+\sum_{2 \leq l<i} w\left(e_{l}\right)
$$

i.e. we have to use agents to block all edges to previously cleared subtrees and then use agents to clear the subtree rooted in $v_{i}$. The label on $e$ hence becomes:

$$
\lambda_{v_{x}}(e)=\max \left\{s\left(v_{y}\right), \max _{i=2, \ldots, m}\left\{c\left(v_{i}\right)\right\}\right\} .
$$

The order defined by $\rho_{i}$ minimizes this term. Once all labels are computed we can find a strategy to clear $G_{T}$ from a vertex $v \in V$ with neighbors $v_{1}, \ldots, v_{m}$ by considering:

$$
a g(v)=\max \left\{s(v), \max _{i=1, \ldots, m}\left\{c_{a g}\left(v_{i}\right)\right\}\right\},
$$

where $c_{a g}\left(v_{i}\right)=\lambda_{v}\left(e_{i}\right)+\sum_{1 \leq l<i} w\left(e_{i}\right)$ similar to $c\left(v_{i}\right)$, but including all neighbors since we do not enter from another vertex when we start the clearing from $v$ directly. To find the minimal strategy we simply compute all labels and then select the vertex where $a g(v)$ is minimal. The resulting strategies are non-contiguous and not optimal. In fig. 2 the execution of a non-contiguous strategy based on the presented labels is illustrated. In [1] Barriere provides details for computing labels for a similar labeling mechanism in $O(n)$, where $n$ is the number of vertices in the tree.

2) Contiguous labels: The contiguous variant of these labels is the basis of the contiguous algorithm. The key difference is that the contiguous strategy first clears $v_{y}$ and then descends into the subtrees. It is motivated by the study of contiguous edge-search strategies for weighted trees by Barriere in [1]. Since we first clear $v_{y}$, all edges to vertices $v_{2}, \ldots, v_{m}$ have to remain blocked after safely clearing $v_{y}$. This means a reversal in the order in which we clear these vertices. Furthermore, when entering the subtree rooted in $v_{i}$ we have the edge to $v_{i}$ already blocked, contrary to the non-contiguous strategy. But the next step is to clear $v_{i}$ itself before descending into the other subtrees. Figure 2 illustrates the difference between the contiguous and non-contiguous strategies. As we are using $s\left(v_{i}\right)$ agents for clearing $v_{i}$ and also block $e$ during this operation we can also take $\sum_{2 \leq l<i} w\left(e_{l}\right)$ as the additional number of agents to guard edges to contaminated neighbors rather than $\sum_{2 \leq l \leq i} w\left(e_{l}\right)$ as done in [8]. Once vertex $v_{i}$ is cleared the block on $e_{i}$ is removed and the term $\sum_{2<l<i} w\left(e_{l}\right)$ remains the maximum number of agents used. Using this perspective it becomes apparent that contiguous and non-contiguous labels actually have the same equations complementing a lemma from [8] that the number of agent needed for a strategy based on noncontiguous labels is equal or better than contiguous labels and showing that the number of agents is indeed equal. In fig. 2 this becomes clearly visible and we therefore refrain from presenting a formal proof.

\section{HYBRID STRATEGIES}

In [8] it was proposed to combine the two current algorithms by separating the neighboring vertices into two sets and clearing one using the contiguous and one with the noncontiguous algorithm. More precisely, for $v_{y}$, coming from $v_{x}$, we seek to partition the neighbors $V:=\left\{v_{2}, \ldots, v_{m}\right\}$ into two sets of vertices $V_{1}$ and $V_{2}$. The first set $V_{1}$ will be cleared with the non-contiguous algorithm. Once all elements of $V_{1}$ are cleared the team clears $v_{y}$ and then proceeds to clear $V_{2}$ with the contiguous algorithm. We thereby divide the weight of the term $\sum_{2 \leq l<i} w\left(e_{l}\right)$ from equation 3 onto two sets. This can greatly reduce the total number of agents 

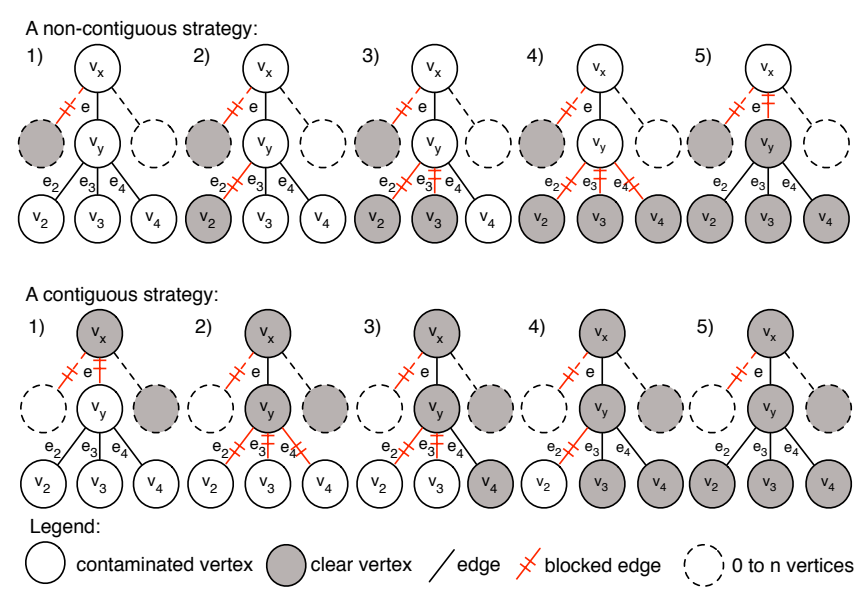

Fig. 2. Illustration of a contiguous and a non-contiguous strategy.

needed. Figure 3 illustrates how such a hybrid strategy would be executed.

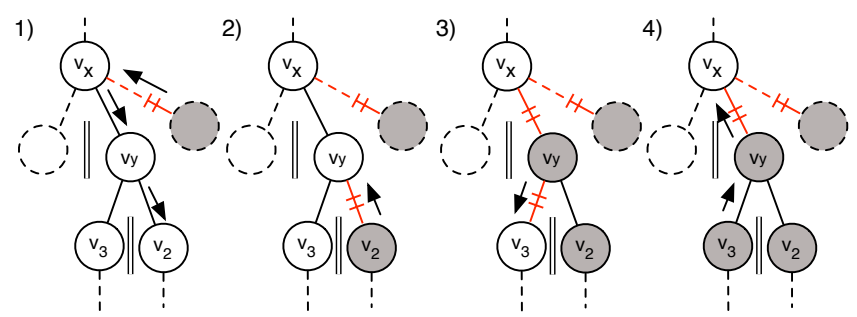

Fig. 3. Execution of the hybrid strategy.

From fig. 3 one complication becomes apparent. Let $V_{1}^{x}$ and $V_{2}^{x}$ be the partitioning of the neighbors of $v_{x}$ when coming from yet another vertex $v_{z}$. If $v_{y} \in V_{1}^{x}$, then $e$ is not blocked when the team enters $v_{y}$, as seen in fig. 3. Once we clear $v_{y}$ we have to add a block on $e$ which increases the total number of agents needed while clearing $V_{2}$, as seen in steps 3 to 5 in fig. 3 . If $v_{y} \in V_{2}^{x}$, then the situation is reversed and we have to add a block on $w(e)$ only while we clear $V_{1}$ and not while clearing $V_{2}$.

Let us denote the case when $v \in V_{1}^{x}$ as case 1 and $v \in V_{2}^{x}$ as case 2 . We can compute a label for both cases, using the superscripts ${ }^{1}$ and ${ }^{2}$. So the labels on edge $e$ become:

$$
\begin{aligned}
h_{u}^{1}\left(V_{1}, V_{2}\right) & =\max \left\{\max _{v_{i} \in V_{1}}\left\{c^{1}\left(v_{i}\right)\right\}, \max _{v_{i} \in V_{2}}\left\{c^{2}\left(v_{i}\right)+w(e)\right\}\right\} \\
h_{u}^{2}\left(V_{1}, V_{2}\right) & =\max \left\{\max _{v_{i} \in V_{1}}\left\{c^{1}\left(v_{i}\right)+w(e)\right\}, \max _{v_{i} \in V_{2}}\left\{c^{2}\left(v_{i}\right)\right\}\right\} \\
\lambda_{v_{x}}^{1}(e) & =\max \left\{s\left(v_{y}\right), \min _{V_{1}, V_{2}}\left\{h_{u}^{1}\left(V_{1}, V_{2}\right)\right\}\right\} \\
\lambda_{v_{x}}^{2}(e) & =\max \left\{s\left(v_{y}\right), \min _{V_{1}, V_{2}}\left\{h_{u}^{2}\left(V_{1}, V_{2}\right)\right\}\right\}
\end{aligned}
$$

where $c\left(v_{i}\right)^{j}=\lambda_{v_{y}}^{j}\left(e_{i}\right)+\sum_{v_{l} \in V_{j}, 2<l<i} w\left(e_{l}\right)$ for $j=1,2$. It is easy to see, however, that $h_{u}^{1}\left(V_{1}, V_{2}\right)=h_{u}^{2}\left(V_{2}, V_{1}\right)$ given that $\lambda_{v_{y}}^{1}\left(e_{i}\right)=\lambda_{v_{y}}^{2}\left(e_{i}\right)$, which is the case since we compute the labels from the leaves upward and these equations are identical. It is however, important to note that the partition still has take into account the penalty term $w(e)$, i.e. only to which side it is assigned is not relevant. Hence, to simplify notation, we will drop superscripts ${ }^{1}$ and ${ }^{2}$. The problem now states as follows:

Definition 4 (Hybrid algorithm: optimal partition):

Given $v_{x}, v_{y}$ and neighbors $V=\left\{v_{2}, \ldots, v_{m}\right\}$ as before find a partition of $V$ into $V_{1}$ and $V_{2}$ s.t. $h_{u}\left(V_{1}, V_{2}\right)$ is minimal.

The proposed algorithm to find partitions will be based on theoretical framework of the next two subsections. First we introduce the concept of batches which cluster vertices and then proceed by developing criteria for optimal partitions into $V_{1}$ and $V_{2}$ in section IV-B. On the basis of this we will develop an algorithm in section IV-C.

\section{A. Batches}

The following will be useful to describe at which vertex within a set $V$ the number of agents is maximal. The proofs are left out here and can found in [?]. We shall call a set of all vertices with $\rho_{i}=a-p$ a batch $B_{p}$, where $a:=\max \left\{\lambda_{v_{y}}\left(e_{i}\right)\right\}$. The set $V$ can have at most $a-1$ batches, i.e. $B_{1}, B_{2}, \ldots, B_{a-1}$. During the execution of a strategy $S$ in the non-contiguous variant we clear the batches in sequence $B_{1}, B_{2}, \ldots, B_{a-1}$ and then clear $v$. For the contiguous variant the order of clearing is reversed. Define the weight of a batch as $w\left(B_{p}\right):=\sum_{v_{i} \in B_{p}} w\left(e_{i}\right)$ and write $w\left(B_{p<k}\right):=\sum_{p<k} w\left(B_{p}\right)$. Define the maximum cost within $V$ to be $h:=\max _{2 \leq i \leq m}\left\{c\left(v_{i}\right)\right\}$ and let $v_{q}$ be a vertex that assumes this maximum, i.e. $h=c\left(v_{q}\right)$, s.t. $v_{q} \in B_{k}$ with $k$ being the largest such possible batch index. Using this notation we can rewrite the maximum cost to be:

$$
\begin{aligned}
h & =w\left(B_{i<k}\right)+w\left(B_{k}\right)-w\left(e_{q}\right)+\lambda\left(e_{q}\right) \\
& =w\left(B_{i \leq k}\right)+\rho_{q}=w\left(B_{i \leq k}\right)+a-k .
\end{aligned}
$$

The following lemma will be relevant for our further results.

Lemma 1: Let $v_{q}$ and $B_{k}$ be as before. Consider any nonempty $B_{k^{\prime}}$ s.t. $k \neq k^{\prime}$. If $k>k^{\prime}$, then $k-k^{\prime} \leq w\left(B_{k^{\prime}<i \leq k}\right)$. Otherwise if $k<k^{\prime}$, then $w\left(B_{k<i \leq k^{\prime}}\right) \leq k^{\prime}-k$.

\section{B. Criteria for optimal partitions}

All vertices in batches $B_{i}$, for $i>k$, do not contribute to the maximum, i.e. a removal of these vertices does not change the maximum cost. We shall call such vertices the tail $T:=\bigcup_{i>k} B_{i}$ of $V$. Their joint weight shall be denoted by $w(T)=\sum_{v_{i} \in T} w\left(e_{i}\right)$. As a consequence of lemma 1 we have $w\left(T_{t}\right)<a-k$.

When partitioning $V$ into $V_{1}$ and $V_{2}$ we shall write $B_{i, 1}$, $B_{i, 2}$ for the batches of $V_{1}$ and $V_{2}, k_{1}, k_{2}$ for $k, v_{q, 1}, v_{q, 2}$ for $v_{q}, h_{V_{1}}, h_{V_{2}}$ for $h$ and $T_{1}$ and $T_{2}$ for $T$. For notational simplicity we will ignore the penalty term in this section and discuss it thereafter when presenting the partitioning algorithm. Finally, for a partition $V_{1}$ and $V_{2}$ we define a maximization criterion as:

$$
c\left(V_{1}, V_{2}\right):=k_{1}+k_{2}+w\left(T_{1}\right)+w\left(T_{2}\right)-\left|h_{1}-h_{2}\right| .
$$


Definition 5 (Balanced and full partitions): Let $V$ be a set of vertices as before. A partitioning of $V$ into $V_{1}$ and $V_{2}$ is called

- full if $k=k_{1}=k_{2}$,

- balanced if $w\left(B_{i \leq k_{1}, 1}\right)-k_{1}=w\left(B_{i \leq k_{2}, 2}\right)-k_{2}$,

- maximal if for any other partition $V_{1}^{\prime}, V_{2}^{\prime}$ we get that $c\left(V_{1}, V_{2}\right) \geq c\left(V_{1}^{\prime}, V_{2}^{\prime}\right)$.

It is easy to see that a partition that is full and balanced will minimize $h_{u}$ and is therefore optimal. Also any full and balanced partition will be maximal. To show that any maximal partition is optimal we need the following lemma to show that $h_{b}:=w\left(B_{i \leq k}\right) / 2+a-k$ is a lower bound on $h_{u}$.

Lemma 2: Given $V$, with $a$ and $k$ as before and any partition $V_{1}$ and $V_{2}$ we have that:

$$
h_{u} \geq w\left(B_{i \leq k}\right) / 2+a-k=h_{b} .
$$

Proof: Detailed proof found in [9].

For full and balanced partitions we have $h_{u}=h_{b}$. But a full and balanced partition may not exist and hence we have to consider maximal partitions.

Lemma 3: If $V_{1}, V_{2}$ is a maximal partition of $V$, then $h_{u}$ is minimal, i.e. the partition is optimal.

Proof: Detailed proof found in [9].

In colloquial terms, we have to find a partition with the largest $k_{1}, k_{2}$ and large tails $T_{1}, T_{2}$ and with $w\left(B_{i \leq k_{1}, 1}\right)$ roughly equal to $w\left(B_{i \leq k_{2}, 2}\right)$.

\section{The partitioning algorithm}

The algorithm is based on a dynamic programming approach motivated by the relation of the maximization criterion to the subset sum problem, one of the early NPcomplete problems [2]. In short, the subset sum problem is to determine whether a set of integer values contains a subset whose values sum up to some given integer $z$. A dynamic programming algorithm to solve it runs in pseudopolynomial time $O(C n)$ where $C$ is the sum of all members of the set and $n$ is the number of elements. Translated to our case this becomes the problem to determine whether $V$ contains a set of vertices $V_{2}$ s.t. the sum of the weight of their respective edges $w\left(V_{2}\right)$ sums up to $z=\lceil w(V) / 2-w(e) / 2\rceil$. Here $w(e)$ is the penalty term from equation 6. A solution $V_{2}$ would minimize $h_{u}$ given that $V_{1}=V \backslash V_{2}, V_{2}$ is a full partition, i.e. it satisfies $k_{1}=k_{2}=k$. Obviously, using the dynamic programming approach for solving the subset sum problem gives no guarantee that $k_{1}=k_{2}=k$. In fact, such a partition may not even exist. The following will be concerned with an algorithm that guarantees to find a full partition if one exists.

Let $A$ be a table with $m-1$ rows and $z=\lceil w(V) / 2-$ $w(e) / 2\rceil$ columns. Set $A(0, j):=0, \forall j$ and $A(i, 0):=0, \forall i$. Each row represents a vertex and they shall be ordered as $v_{m}, \ldots, v_{2}$, i.e. $v_{m}$ corresponds to row one, $v_{m-1}$ to row two and so on. Write $c_{i}$ for $w\left(e_{m-i+1}\right)$, i.e. the cost added to $V_{2}$ by adding the vertex in row $i$. If $c_{i}>j$, then $A(i, j)=$ $A(i-1, j)$, otherwise $A(i, j)=\max \{A(i-1, j), A(i-1, j-$ $\left.\left.\left.c_{i}\right)+c_{i}\right)\right\}$. An entry $A(i, j)$ in the table is then the maximal weight for $V_{2}$ achievable using vertices $v_{m}, \ldots, v_{m-i+1}$. The table is filled as usual for the subset sum problem. If an entry in $A$ exists s.t. $A(i, j)=\lceil w(V) / 2-w(e) / 2\rceil$, then we have a partition that is optimal w.r.t. to the distribution of the edge weights onto $V_{1}$ and $V_{2}$. This is, however, only one part in the optimization. An entry in $A$ represents possibly multiple partitions, some of which do not satisfy that $k_{1}=k_{2}=k$. A particular partition can be thought of as a path within the table. In [9] examples illustrating this are presented. Finding an optimal partition is hence the problem of finding an entry with $A(i, j)=\lceil w(V) / 2-w(e) / 2\rceil$ for which we have a path that represents a maximal partition. We will show how to compute whether such a path exists for the case of full partitions.

Since we ordered the vertices in reverse order we can view the problem from the perspective of adding vertex by vertex with decreasing index to $V_{2}$ as we proceed through the rows of $A$. For $V_{1}$ we can view it as if we are removing vertices with decreasing index from $V_{1}$. The main question is what happens to $k_{1}$ for $V_{1}$ and $k_{2}$ for $V_{2}$ as we remove and add vertices. When we add a vertex $v \in B_{u, 1}$ from $V_{1}$ to $V_{2}$ we know that all other vertices in $V_{2}$ are in batches $B_{j \geq u, 2}$. Write $V_{1}^{\prime}=V_{1} \backslash\{v\}$ and $V_{2}^{\prime}=V_{2} \cup\{v\}$. Define $S\left(V_{2}\right):=$ $\sum_{1 \leq i \leq k_{2}} w\left(B_{i}, 2\right)$ to be the support of $V_{2}$. Now if $k_{2}-$ $u>S\left(V_{2}\right)$, then $v=v_{q}^{\prime}$ will be the new maximum for $V_{2}^{\prime}$. Otherwise, if $k_{2}-u \leq S\left(V_{2}\right)$, then $v_{q}=v_{q}^{\prime}$. To illustrate this with our example set of vertices simply choose $V_{2}=$ $\left\{v_{9}\right\}$. Clearly $v_{q, 2}=v_{9}$ and $S\left(V_{2}\right)=2$ and adding $v_{5}$ will lead to $v_{q, 2}^{\prime}=v_{5}$. Similarly for $V_{1}^{\prime}$, when removing $v$ with associated edge $e_{v}$, the support will be reduced to $S\left(V_{1}^{\prime}\right)=$ $S\left(V_{1}\right)-w\left(e_{v}\right)$. Now the maximum $v_{q, 1}^{\prime}$ may shift to a vertex of a lower batch if $\exists B_{b}$ s.t. $k_{1}-b>S\left(V_{1}\right)$, otherwise it will remain at it former vertex s.t. $v_{q, 1}^{\prime}=v_{q, 1}$.

As long as $k_{1}=k_{2}=k$ we know that $S\left(V_{1}\right)=$ $w\left(V_{1}\right), S\left(V_{2}\right)=w\left(V_{2}\right), w\left(T_{1}\right)=w\left(T_{2}\right)=0$ and we do not need to keep track of these values. Once we add a vertex $v \in B_{u, 1}$ from $V_{1}$ to $V_{2}$ with $k_{2}-u>S\left(V_{2}\right)$ we will have $k_{2}^{\prime}<k$ and the path will not be a valid solution. Let us define two further tables $K_{1}(i, j)$ and $K_{2}(i, j)$ in which we will keep track of $k_{1}$ and $k_{2}$. For our case the computation of $K_{1}(i, j)$ and $K_{2}(i, j)$ involves only a simple check, whether upon addition/removal of the vertex the current $K_{1}$ and $K_{2}$ can be maintained. If this is not the case we discard the solution path by setting $K_{1}(i, j)=0$ or $K_{2}(i, j)=0$. The pseudo code in 1 shows how to compute $A, K_{1}$ and $K_{2}$. Initially we set $K_{1}(0, j)=K_{2}(0, j)=k$. It is obvious that $k_{1}, k_{2}$ are monotonically decreasing w.r.t. to growing $i, j$, except for the special case for $V_{1}$ if we remove the first vertex $v_{2}$ in the last row of the table and at this point have $v_{q, 1}=v_{2}$ and $B_{b_{1}, 1}=\left\{v_{2}\right\}$, i.e. there is no other vertex in its batch. Dealing with this special case merely complicates notation without changing the methodology and we will therefore ignore it. Now, an entry $A(i, j)=z$ with $K_{1}(i, j)=K_{2}(i, j)=k$ has a path that represents a full and balanced partition which is therefore optimal. If no such entry exists, then neither does a full and balanced partition. In [9] a starting point to construct an analogue algorithm for 


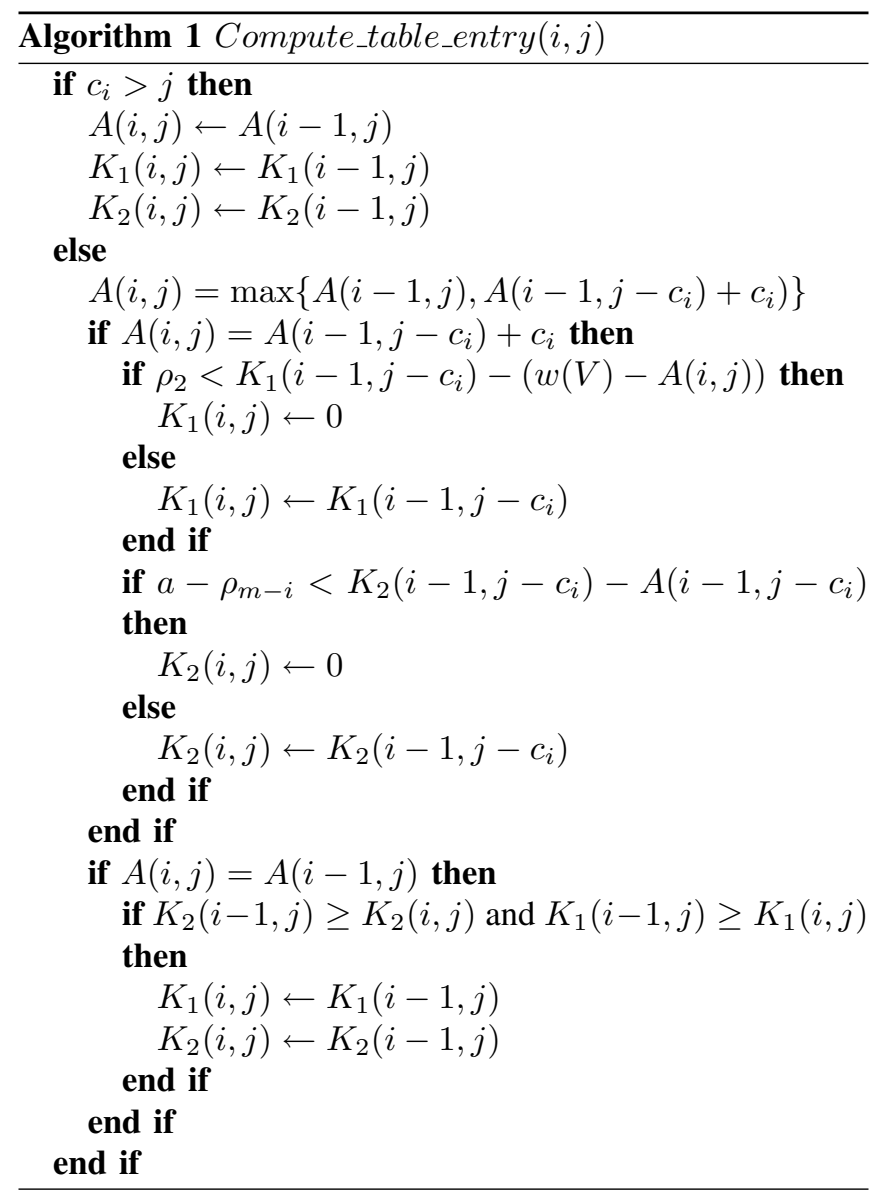

maximal partitions is given.

\section{Discussion AND CONCLUSION}

We presented a new approach for finding strategies for GRAPH-CLEAR in a tree which requires solving a partitioning problem. We presented criteria for optimal partitions and based on these we presented an algorithm that computes optimal partitions given that a full and balanced partition exist. In [9] the open problem to develop to compute maximal partitions is discussed in more detail. Apart from various other details dealt with in [9], the key problem for finding maximal partitions efficiently is to find a way to compute $C(i, j)$ from the entries for $A, K_{1}, K_{2}, T_{1}, T_{2}$ at $(i-1, j)$ and $\left(i-1, j-c_{i}\right)$. We do already know how $K_{1}, K_{2}, T_{1}$ and $T_{2}$ evolve when adding a vertex $v_{m-i+1}$. Hence we can identify whether the path from $(i-1, j)$ or from $\left(i-1, j-c_{i}\right)$ leads to a better partition w.r.t. to $C(i, j)$. The only open problem that remains is whether a path representing an optimal partition at entry $A(i, j)$ will be optimal at all previous entries. In other words, it is the question whether the partition for $A(i, j)$ that maximizes $C(i, j)$ can be computed based on the description of the partitions in the previous row $i-1$.

Even without computing maximal partitions a greedy partition for a hybrid method already outperforms the previous algorithms from [7] and [8]. On the other hand, in a realistic robotic application of GRAPH-CLEAR a reduction by a few robots is already significant decrease in costs and therefore an optimal solution for the partitioning is of interest. Furthermore, from a graph-theoretical perspective the investigation whether optimal solutions for general strategies on trees exist motivates further analysis of the hybrid method. Yet, we believe the current progress for finding GRAPH-CLEAR strategies already gives a good basis for using it in robotic applications for surveillance.

\section{REFERENCES}

[1] L. Barriere, P. Flocchini, P. Fraigniaud, and N. Santoro. Capture of and intruder by mobile agents. In Proceedings of the 14th annual ACM symposium on Parallel algorithms and architectures, pages 200-209, 2002.

[2] M.R Garey and D.S. Johnson. Computers and Intractability. A guide to the theory of NP-Completeness. W.H. Freeman and Company, 1979

[3] B. Gerkey, S. Thrun, and G. Gordon. Visibility-based pursuit-evasion with limited field of view. International Journal of Robotics Research, 25(4):299-316, 2006.

[4] L.J. Guibas, J.-C. Latombe, S.M. LaValle, D. Lin, and R. Motwani. A visibility-based pursuit-evasion problem. International Journal of Computational Geometry and Applications, 9(4/5):471-494, 1999.

[5] A. Kolling and S. Carpin. Multirobot cooperation for surveillance of multiple moving targets - a new behavioral approach. In Proceeding of the IEEE International Conference on Robotics and Automation, pages 1311-1316, 2006.

[6] A. Kolling and S. Carpin. Cooperative observation of multiple moving targets: an algorithm and its formalization. International Journal of Robotics Research, 26(9):935-953, 2007.

[7] A. Kolling and S. Carpin. Detecting intruders in complex environments with limited range mobile sensors. In K. Kowzlowski, editor, Robot Motion and Control 2007, Lecture Notes in Information and Control, pages 417-426. Springer, 2007.

[8] A. Kolling and S. Carpin. The graph-clear problem: definition, theoretical properties and its connections to multirobot aided surveillance. In Proceedings of the IEEE/RSJ International Conference on Intelligent Robots and Systems, pages 1003-1008, 2007.

[9] A. Kolling and S. Carpin. An improved algorithm for the GRAPH SEARCH problem. Technical Report 2007-3, UC Merced, 2007.

[10] N. Megiddo, S.L. Hakimi, M.R. Garey, D.S. Johnson, and C.H Papadimitriou. The complexity of searching a graph. Journal of the ACM, 35(1):18-44, 1988

[11] L. E. Parker. Distributed algorithms for multi-robot observation of multiple moving targets. Autonomous robots, 12(3):231-255, 2002

[12] T. Parsons. Pursuit-evasion in a graph. In Y. Alavi and D. Lick, editors, Theory and Applications of Graphs. Springer-Verlag, 1976.

[13] S. Sachs, S. Rajko, and S. M. LaValle. Visibility-based pursuit-evasion in an unknown planar environment. International Journal of Robotics Research, 23(1):3-26, 2004.

[14] I. Suzuki and M. Yamashita. Searching for a mobile intruder in a polygonal region. SIAM Journal of Computing, 21(5):863-888, 1992. 